\title{
Are we still wondering or wandering in the dark?
}

\author{
Davide Cattano
}

Received: 20 February 2011/Accepted: 4 March 2011/Published online: 17 March 2011

(C) SIMI 2011

Acute renal failure (ARF) or acute kidney injury (AKI), especially superimposed in a chronic renal failure patient can detrimentally and terminally unbalanced a hardly maintained physiologic function, determining the dialysis for that patient. Otherwise, the acute phenomenon, possibly self limited, can acutely affect a relatively healthy patient, as well as compromising the renal function [1].

It is not uncommon as well that the patients with chronic renal failure would also be the one requiring radiology or cardiology studies involving contrast.

Besides several measures have been taken, several strategies and protocols have been applied, the "evidences" are still uncertain. In the age of evidences and certainties, how could we possibly intervene with a clinical common sense?

Contrast-induced nephropathy (CIN) is reported to occur in as many as $14.5 \%$ of unselected patients undergoing coronary angiography/intervention [2], and is the third most common cause of in-hospital acute renal failure after hypotension and surgery [3]. Important risk factors for CIN include pre-existing renal dysfunction, especially that due to diabetic nephropathy, reduced circulating volume, the volume and type of contrast agent employed, and concomitant administration of potentially nephrotoxic drugs $[4,5]$. Several agents have been proposed to provide prophylaxis against CIN [6]. However, until recently, only saline hydration $(1 \mathrm{ml} / \mathrm{kg} 0.45 \%$ saline for $12 \mathrm{~h}$ pre- and

\section{Cattano $(\square)$}

Department of Anesthesiology, The University of Texas Medical

School at Houston, Houston, USA

e-mail: davide.cattano@uth.tmc.edu

D. Cattano

Anesthesia Preoperative Clinic Memorial Hermann Hospital

Texas Medical Center, Houston, USA post-contrast exposure has been confirmed to be effective) [5].

The precise mechanisms leading to CIN remain a matter of debate, although the root of the problem appears to be an injury to the renal medulla resulting from a combination of reduced blood flow, an osmotic effect, and direct tubular toxicity $[7,8]$. The last of these may be a direct result of toxic free radical release, which occurs after contrast administration.

Acetylcysteine (NAC), an antioxidant, has been advocated for a while in addition to saline hydration in preventing CIN $[9,10]$, although this has not been a universal finding [11, 12]. Successful protocols tested to-date require the initiation of therapy on the day before contrast exposure, precluding the treatment of same-day and emergency patients.

However, different protocols and administration routes have been used, but systematic reviews failed to find a definitive answer whether the practice is effective to be justified. While the recommendations of using NAC and ascorbic acid are based on the proposed lack of evidenced efficacy that may be limited to the high risk population only. The management is clinically simple and could still present a benefit without any evident safety concern for the patients.

In the current study of Calabro et al. [13], investigators found a reduction in the incidence of CIN, confirming previous results of a high risk population [14] in the low risk patients (no diabetes, age $<65$ and no creatinine greater than $1.4 \mathrm{mg} / \mathrm{dL}$ ).

Raw numbers for their studies are, respectively, $6.7 \%$ in the initial retrospective study, an with incidence of CIN of $4.4 \%$ in the treatment high risk group versus $9.8 \%$ in the non-treated low risk group, and $7.1 \%$ in the prospective observational study using a historical control, with an 
incidence of $2.6 \%$ in the low risks treated group versus $11.2 \%$ in the non-treated. Whether or not we can argue that the studies were not randomized, were not blinded and were not controlled, still the differences are clinically relevant.

Moreover, the main point remain the identification of risk factors that can easily being modified by preoperative or pre-procedural protocols involving suspension of diuretics, hypotensive drugs affecting renal perfusion and providing good hydration, underestimated probably by most clinicians. As shown by Baker [5], a rapid infusion protocol can still be applied wherever a missed preparation has occurred and could substitute the oral preparation.

Should we proceed with a treatment that is doubtful in its clinically efficacy? Well, we can at least assume it is safe and relatively inexpensive. Moreover, as Calabro points out, the reported incidence of CIN is still ranging between 2 and $50 \%$, meaning a difficulty either to identify, or diagnose or stratify the patients at risk for it, and in consideration of different patients' response. Indeed, the injury seems to be unrelated to the severity of the chronic renal condition or the contrast used [1]. One paradigm could be to utilize new (because proposed as diagnostic tools) markers of injury and possibly more sensitive and specific tests, to support and identify earlier the renal injury or to anticipate the patient risk score based on those parameters [15].

The work by Calabro and associates is an interesting result and should not prompt any further doubt other then to continue and prophylactively intervene in the cath laboratory and any radiology suites protecting them from an impending and potential nephropathy, as well possibly utilize NAC in the operating room, considering large prospective randomized studies with better standardized and stratified patient cohorts.

Conflict of interest None.

\section{References}

1. Birck R, Krzossok S, Markowetz F, Schnülle P, van der Woude FJ, Braun C (2003) Acetylcysteine for prevention of contrast nephropathy: meta-analysis. Lancet 362(9384):598-603
2. McCullough PA, Wolyn R, Rocher LL, Levin RN, O'Neill WW (1997) Acute renal failure after coronary intervention: incidence, risk factors, and relationship to mortality. Am J Med 103:368-375

3. Hou SH, Bushinsky DA, Wish JB, Cohen JJ, Harrington JT (1983) Hospital-acquired renal insufficiency: a prospective study. Am J Med 74:243-248

4. James MT, Ghali WA, Tonelli M, Faris P, Knudtson ML, Pannu N, Klarenbach SW, Manns BJ, Hemmelgarn BR (2010) Acute kidney injury following coronary angiography is associated with a long-term decline in kidney function. Kidney Int 78(8):803-809

5. Baker CS, Baker LR (2001) Prevention of contrast nephropathy after cardiac catheterisation. Heart 85:361-362

6. Pannu N, Wiebe N, Tonelli M (2006) Alberta kidney disease network prophylaxis strategies for contrast-induced nephropathy. JAMA 295(23):2765-2779

7. Briguori C, Quintavalle C, De Micco F, Condorelli G (2010) Nephrotoxicity of contrast media and protective effects of acetylcysteine. Arch Toxicol [Epub ahead of print]

8. Bakris GL, Lass N, Gaber AO, Jones JD, Burnett JC Jr (1990) Radiocontrast medium-induced declines in renal function: a role for oxygen free radicals. Am J Physiol 258:F115-F120

9. Tepel M, van der Giet M, Schwarzfeld C, Laufer U, Liermann D, Zidek W (2000) Prevention of radiographic-contrastagentinduced reductions in renal function by acetylcysteine. N Engl J Med 343:180-184

10. Pannu N, Manns B, Lee H, Tonelli M (2004) Systematic review of the impact of $N$-acetylcysteine on contrast nephropathy. Kidney Int 65(4):1366-1374

11. Briguori C, Manganelli F, Scarpato P et al (2002) Acetylcysteine and contrast agent-associated nephrotoxicity. J Am Coll Cardiol 40:298-303

12. Allaqaband S, Tumuluri R, Malik AM et al (2002) Prospective randomized study of $\mathrm{N}$-acetylcysteine, fenoldopam, and saline for prevention of radiocontrast-induced nephropathy. Catheter Cardiovasc Interv 57:279-283

13. Calabrò $P$, Bianchi R, Crisci M, Caprile M, Bigazzi MC, Palmieri R, Golia E, De Vita A, Romano IJ, Limongelli G, Russo MG, Calabrò R (2011) Use and efficacy of saline hydration and $\mathrm{N}$ acetyl cysteine to prevent contrast-induced nephropathy in lowrisk populations undergoing coronary artery angiography. Intern Emerg Med. doi:10.1007/s11739-011-0513-y

14. Calabrò P, Bianchi R, Caprile M, Sordelli C, Cappelli Bigazzi M, Palmieri R, Gigantino G, Limongelli G, Capozzi G, Cuomo S, Calabrò $\mathrm{R}$ (2010) Use of $\mathrm{NaCl}$ saline hydration and $\mathrm{N}$-acetyl cysteine to prevent contrast induced nephropathy in different populations of patients at high and low risk undergoing coronary artery angiography. Min Cardioangiol 58(1):35-40

15. Darmon M, Schortgen F, Vargas F, Liazydi A, Schlemmer B, Brun-Buisson C, Brochard L (2010) Diagnostic accuracy of Doppler renal resistive index for reversibility of acute kidney injury in critically ill patients. Intensive Care Med S1432-S1238 